\title{
Reconstrucción de Fracturas Orbitarias Utilizando Tecnología Digital Tridimensional: Una forma de Optimizar Resultados
}

\author{
Orbital Fracture Reconstruction Using Digital Three-Dimensional \\ Technology: A Way to Optimize Results
}

Rubén Soto Galaz'; Mónica Astudillo Ramírez; Rodrigo Bravo Ahumada ${ }^{1,2}$ \& Renato Gunckel Muñoz ${ }^{1,2}$

SOTO, G. R.; ASTUDILLO, R. M.; BRAVO, A. R. \& GUNCKEL, M. R. Reconstrucción de fracturas orbitarias utilizando tecnología digital tridimensional: Una forma de optimizar resultados. Int. J. Odontostomat., 15(3):782-787, 2021.

RESUMEN: La reconstrucción de las paredes orbitarias fracturadas es compleja debido a la gran cantidad de parámetros volumétricos que posee.Una restitución inadecuada de ellas habitualmente está asociada a secuelas postquirúrgicas en el paciente. El contar con herramientas que optimicen la restitución de la forma anatómica de la órbita en su reconstrucción es de vital importancia, y la utilización de nuevas tecnologías ha permitido mejorar los resultados quirúrgicos, tanto anatómicos como funcionales. El objetivo de este artículo es mostrar dos herramientas quirúrgicas que permiten optimizar los resultados terapéuticos en pacientes con fractura de órbita, que son el modelo estereolitográfico con imagen en espejo y la tomografía computada intraoperatoria. Se presentan las características de estas herramientas, su utilización en tres casos de pacientes con fractura orbitaria y los resultados obtenidos en el post operatorio.

PALABRAS CLAVE: fractura orbitaria, impresión tridimensional (impresión 3d), estereolitografía, tomografía computada.

\section{INTRODUCCIÓN}

La reconstrucción de las paredes orbitarias fracturadas es un tratamiento complejo y requiere de un manejo óptimo para evitar secuelas posteriores. Esta reconstrucción es compleja ya que la fosa orbitaria es una unidad anatómica que está conformada por 4 paredes óseas de distintas características, asociada a estructuras musculares y neurológicas que participan activamente en la funcionalidad del globo ocular.

Las fracturas orbitarias (FO) habitualmente comprometen y modifican los puntos de referencia utilizados para una buena restitución de la anatomía, y la instalación de elementos protésicos como mallas de titanio $u$ otros pueden instalarse en una posición no óptima, generando, en algunos casos, secuelas funcionales en el paciente como enoftalmos, exoftalmos o diplopía (Bell \& Markiewicz, 2009; Shaye et al., 2015).

El uso de tecnología digital como la tomografía computarizada (TC), archivos DICOM, archivos STL y la impresión 3D, permite una mejor visualización de las estructuras afectadas. Esta tecnología ha ayudado significativamente a precisar el diagnóstico y planificar terapias reconstructivas en ortopedia y cirugía maxilofacial. Específicamente, en cirugía maxilofacial su uso ha sido principalmente en planificación quirúrgica digital 3D en deformidades y malformaciones faciales, la impresión de guías quirúrgicas y modelos estereolitográficos, impresión de elementos protésicos, y estudios de navegación intraoperatoria entre otros (Ghai et al., 2018). De ahí la importancia de apoyarse en nuevas herramientas

\footnotetext{
${ }^{1}$ Equipo de Cirugía y Traumatología Bucal y Maxilofacial, Servicio de Cirugía, Hospital San José, Santiago, Chile.

${ }^{2}$ Departamento de Cirugía y Traumatología Bucal y Maxilofacial, Facultad de Odontología, Universidad de Chile, Santiago, Chile.
} 


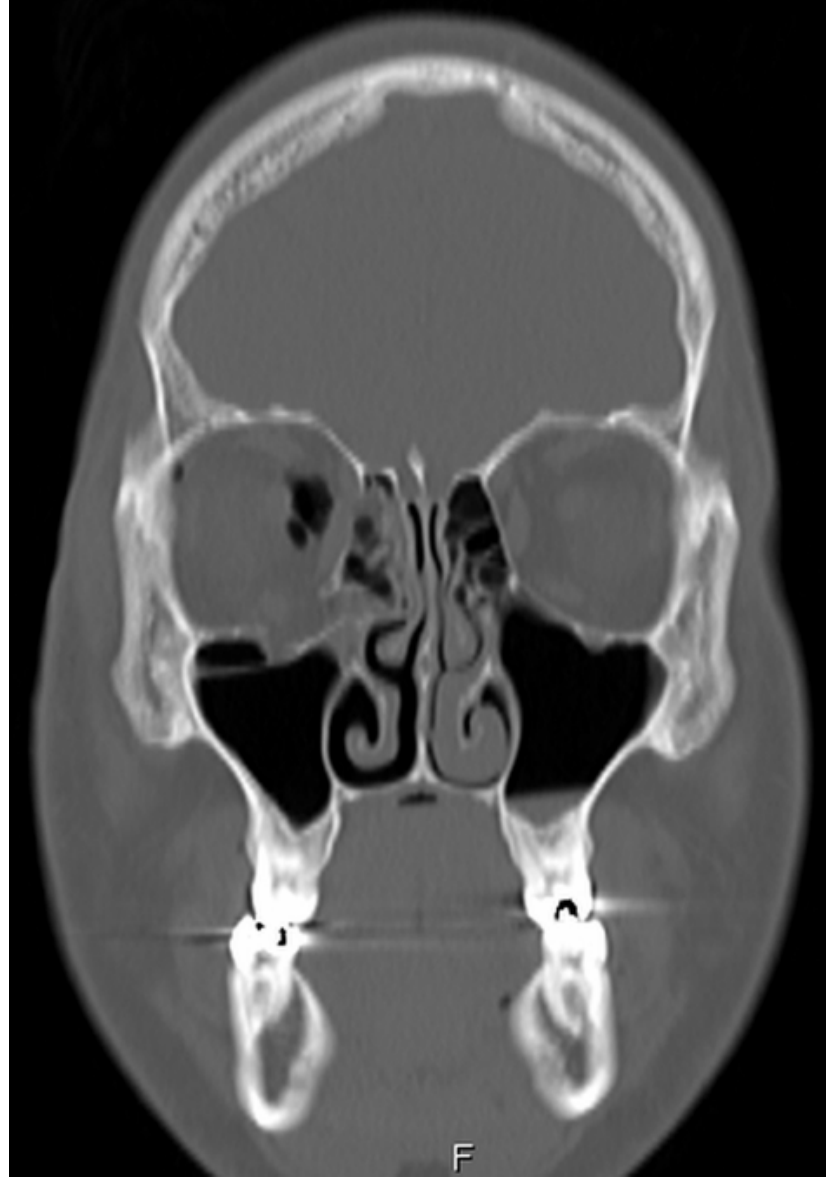

Fig. 1. TC preoperatoria. Se evidencia fractura de piso orbitario derecho con desplazamiento de tejidos a seno maxilar. tecnológicas a manera de optimizar los resultados y reducir la morbilidad en los pacientes con FO.

En este artículo, se describen tres casos de FO resueltos con ayuda de dos herramientas tecnológicas de apoyo en el tratamiento quirúrgico de las fracturas de paredes orbitarias, una es la utilización de modelos estereolitográficos (ME) con imagen en espejo y la segunda es la utilización de tomografía computarizada intraoperatoria.

\section{REPORTE DE CASO}

Caso 1. Paciente masculino de 22 años, ingresa con antecedentes de trauma facial. Al examen clínico presenta hematoma y equimosis periorbitaria derecha, sin alteraciones mayores en oculomotilidad, y presencia de hemorragia subconjuntival en bulbo ocular derecho. $\mathrm{Pa}$ ciente refiere de diplopía en supraducción. En TC del macizo craneofacial se observa una fractura de piso orbitario ipsilateral sin complicación intracraneana (Fig. 1).

El tratamiento propuesto fue la instalación de malla de titanio preformada en el defecto óseo, adaptada previamente en modelo estereolitográfico de órbita con imagen en espejo (Fig. 2a,b). En TC de control se observó el adecuado posicionamiento de la malla (Fig. 3). En el primer control, a una semana de la cirugía, paciente no presentaba dolor, refiriendo mejora importante en

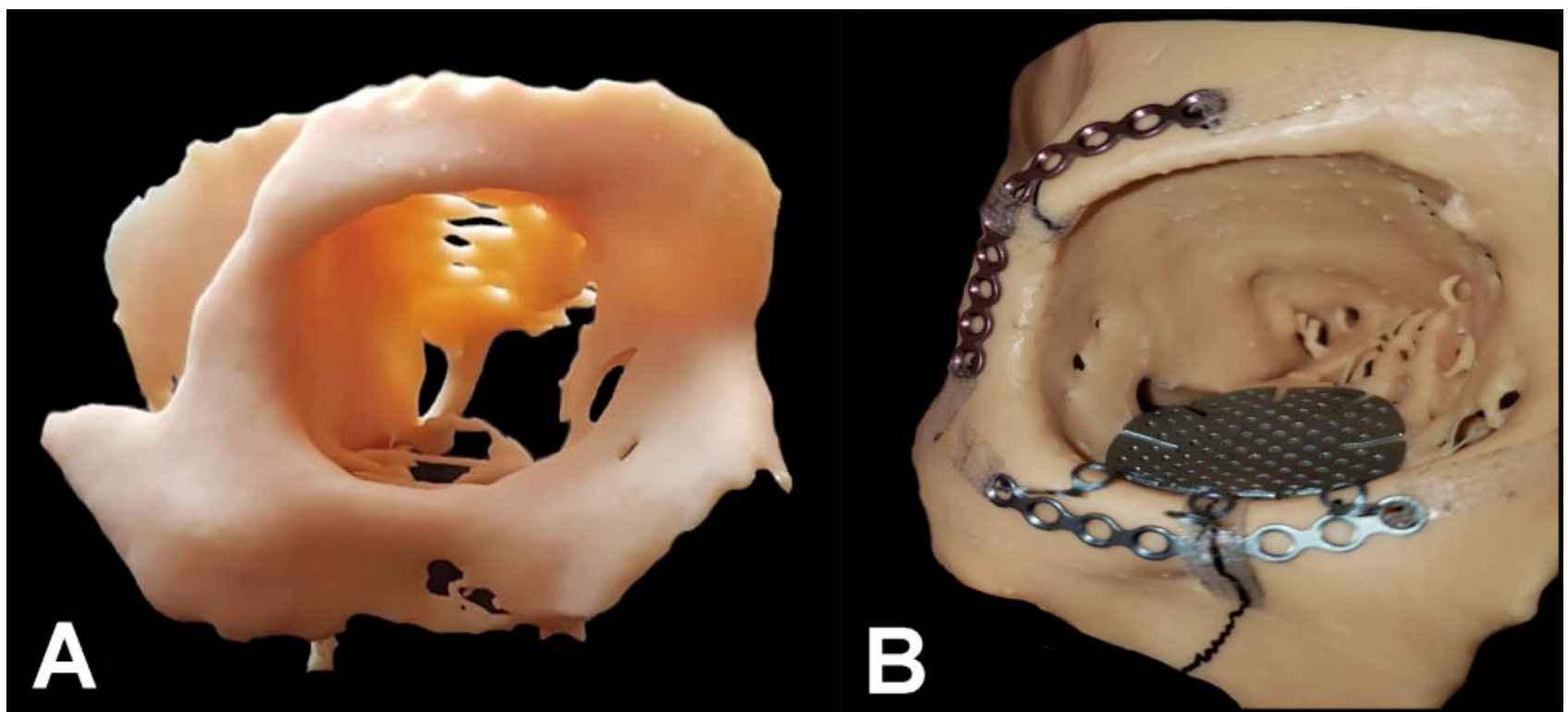

Fig. 2. Modelo estereolitográfico (A) Órbita derecha con imagen en espejo de órbita sana. (B) Malla de titanio preformada y ajustada en modelo estereolitográfico. 
diplopía. En su segundo control a un mes de la cirugía paciente se encontraba en franca mejoría y avanzó mejoría en diplopía. Se envió el paciente a tratamiento complementario con kinesiólogo para mejorar resultados.

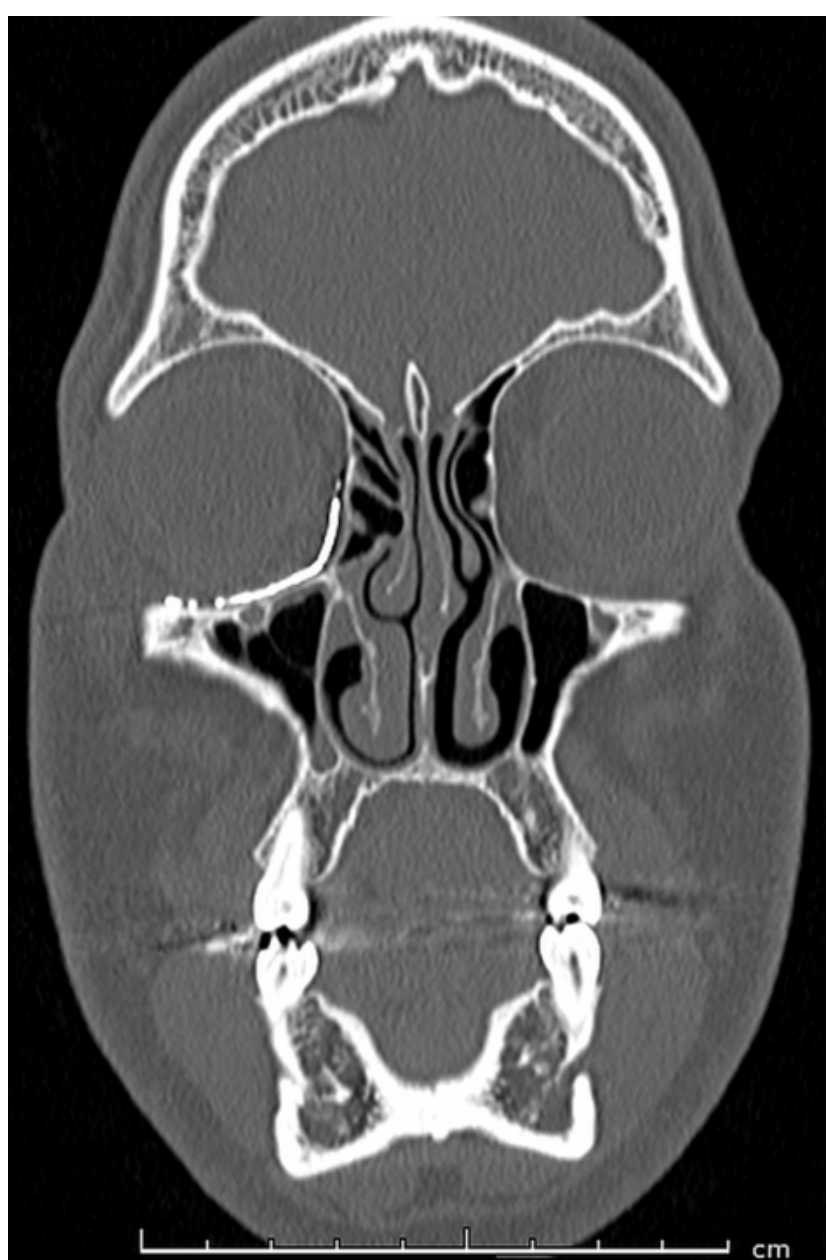

Fig. 3. TC de control. Se observa adecuado posicionamiento de malla, reconstituyendo anatomía de cono orbitario.

Caso 2. Paciente masculino de 28 años, sufrió caída de altura de aproximadamente 10 metros, cursando con TEC complicado, fractura de seno frontal y fractura de piso de órbita derecha, la cual se evidenció en TC (Fig. 4a,b). Fue hospitalizado y operado por servicio de neurocirugía sin incidentes. Al momento de la evaluación maxilofacial, no indicó dolor ni diplopía, presentó oculo motilidad conservada, pero cursó con enoftalmos significativo, por lo que se programó cirugía post alta de servicio de neurocirugía. Se solicitó modelo estereolitográfico con imagen en espejo de la órbita sana para moldeado de malla de titanio (Fig. 5). La cirugía finalmente se realizó luego de un mes de ocurrido el trauma aproximadamente. Al momento de la cirugía se evidenció fibrosis de los tejidos desplaza- dos al seno maxilar, sin embargo, se pudo liberar tejido muscular y la malla premoldeada se pudo instalar sin mayores complicaciones. Se corroboró el adecuado posicionamiento de la malla mediante TC (Fig. 6a,b). En el control inmediato paciente indicó no tener alteraciones visuales. Auna semana de la cirugía, paciente relató diplopía en supraducción, la cual se mantuvo sin mejoría en controles posteriores, determinando la necesidad de evaluación por servicio de oftalmología.

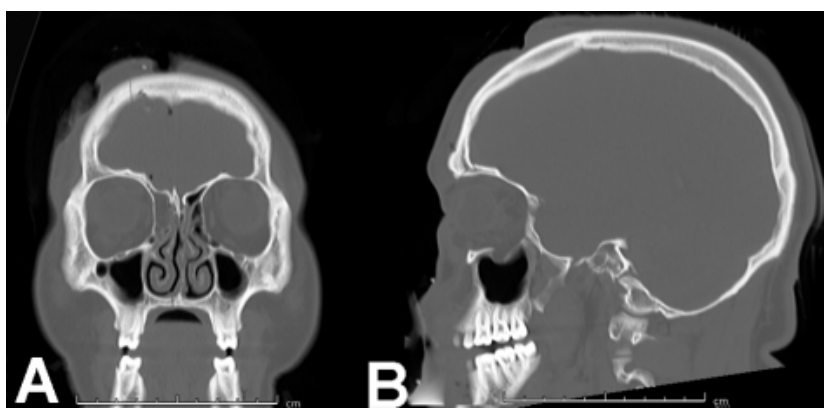

Fig. 4. TC preoperatoria. Se observa fractura de piso orbitario de la órbita derecha con desplazamiento de tejidos hacia seno maxilar. (A) Corte coronal. (B) Corte sagital.

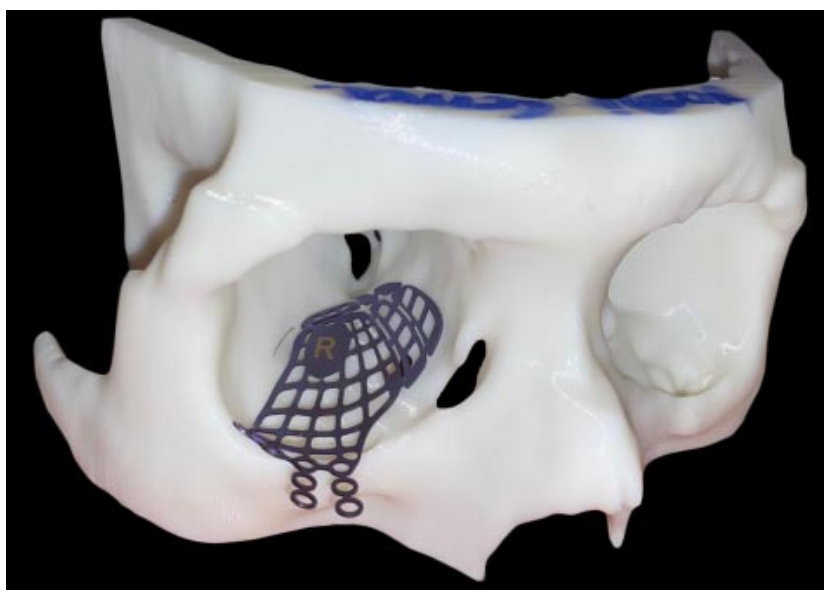

Fig. 5. Modelo estereolitográfico con imagen en espejo, en el que órbita izquierda es reflejada en órbita derecha. Se observa malla de titanio ajustada en modelo.

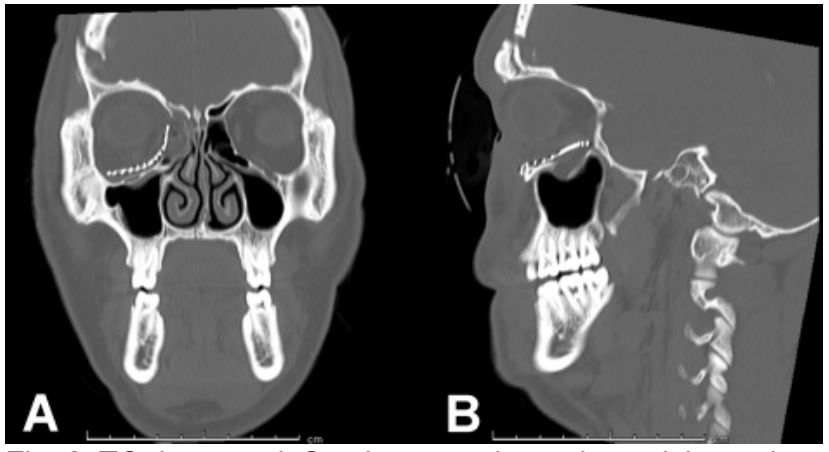

Fig. 6. TC de control. Se observa adecuado posicionamiento de malla protésica y remanencia de tejidos proyectados a seno maxilar. (A) Corte coronal. (B) Corte sagital. 
Caso 3. Paciente femenino de 16 años, con antecedentes de trauma facial, consultó por sensación de disestesia en región hemifacial izquierda y diplopía. En el examen físico se observó edema periorbitario con equimosis, asociados a herida contusa en región geniana ipsilateral, y enoftalmos. En TC se evidenció fractura de piso orbitario izquierdo (Fig. 7), con gran desplazamiento de tejidos blandos hacia seno maxilar.

El tratamiento quirúrgico propuesto fue la reconstrucción con malla de titanio preformada y adap-

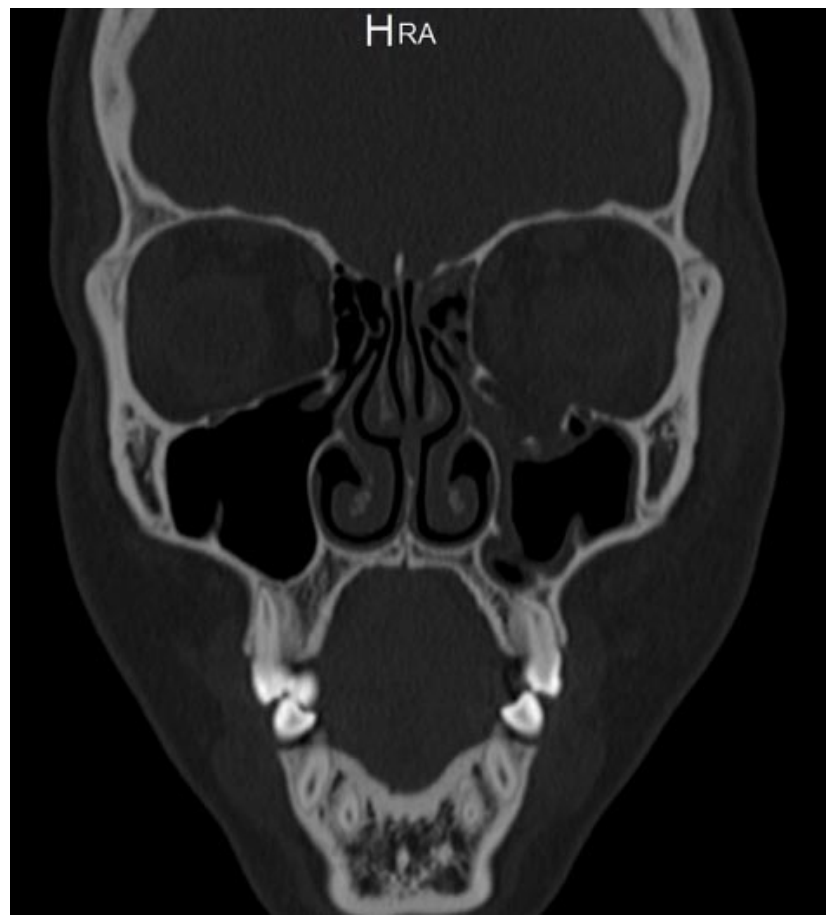

Fig. 7. TC preoperatoria. Se observa fractura de piso orbitario izquierdo con desplazamiento de tejidos hacia seno maxilar. tada en un modelo estereolitográfico de la zona afectada. Durante el procedimiento se realizó TC intraoperatoria (Fig. 8), la que evidenció el correcto posicionamiento tridimensional de la malla (Fig. 9ac). En el primer control postoperatorio, luego de 6 días, persistió la diplopía en todos los movimientos, aparentemente por causa inflamatoria, y disestesia en evolución favorable. Al mes de la cirugía, persistió la diplopía en infraducción. Tres meses después de la cirugía, presentó sólo diplopía en infraducción y hubo resolución completa de disestesia. Dado lo anterior, la paciente fue derivada para evaluación por servicio de oftalmología.

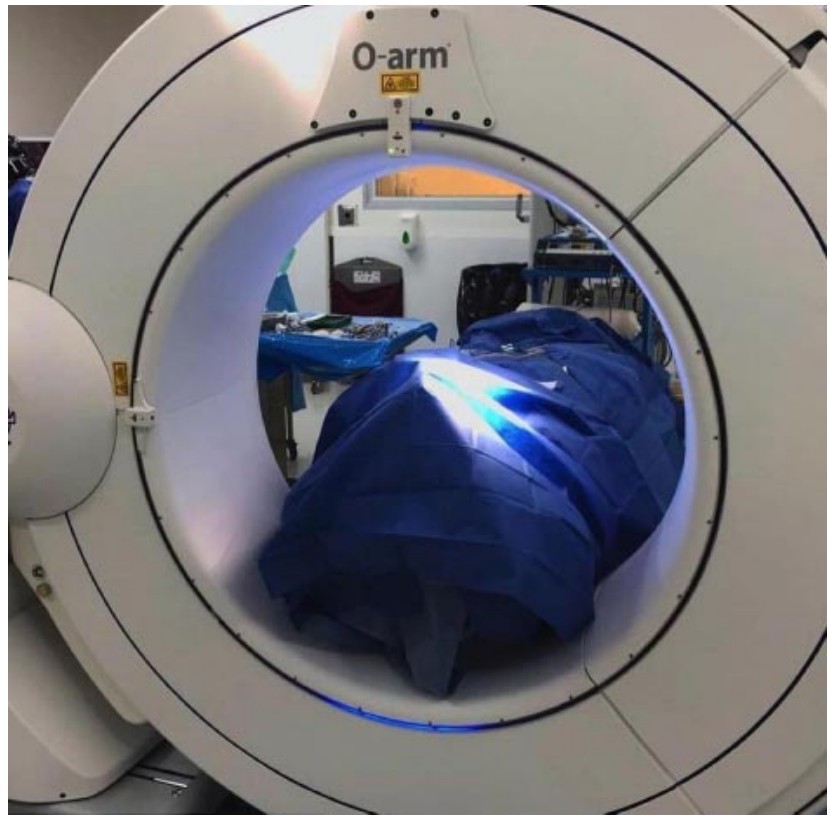

Fig. 8. Sistema de tomografía computarizada intraoperatoria móvil.

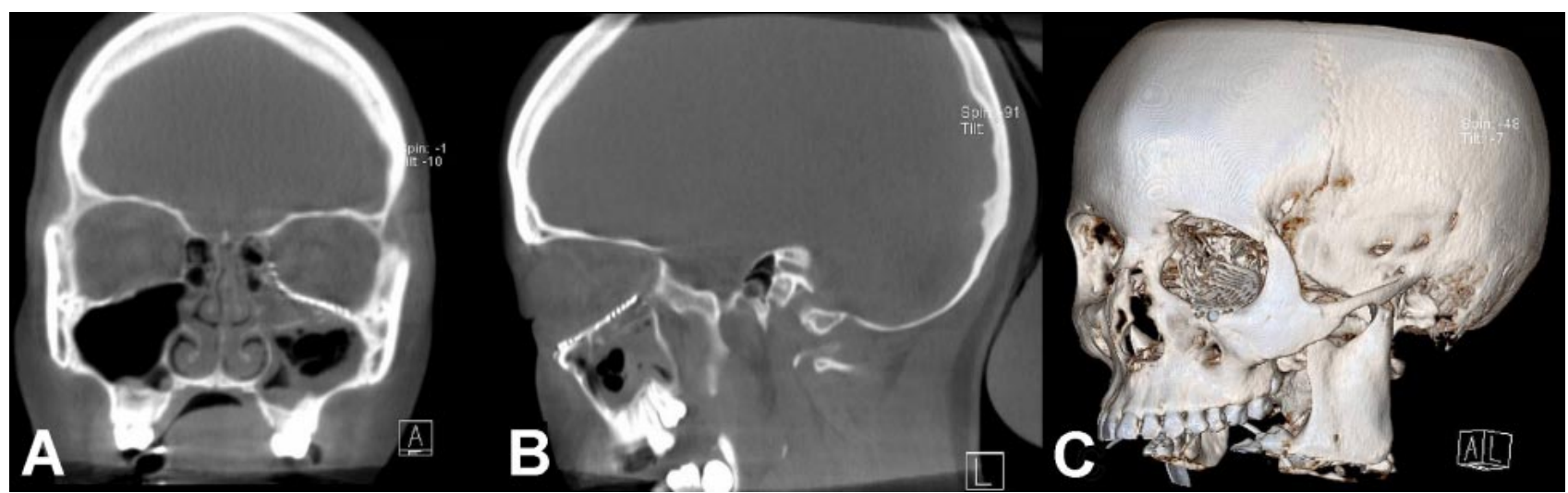

Fig. 9. TC intraoperatoria confirma adecuado posicionamiento de malla protésica sobre zona de fractura. (A) Corte coronal. (B) Corte sagital. (C) Reconstrucción tridimensional. 


\section{DISCUSIÓN}

El piso orbitario es una estructura frágil, la cual puede verse comprometida en traumas de la región maxilofacial, lo que podría traducirse en su fractura y consecuente colapso. La fractura de piso orbitario representa alrededor de un $10 \%$ de todas las fracturas faciales, comprendiendo entre $30 \%$ a $40 \%$ de las fracturas que implican a la órbita (Bell \& Markiewicz; Warwar et al., 2000).

Algunas de las indicaciones de tratamiento quirúrgico de estas fracturas son: presencia de enoftalmos $(2 \mathrm{~mm})$, disfunción en la movilidad ocular, diplopía persistente y respuesta alterada ante test de ducción forzada. Se consideran también como indicación los hallazgos de pinzamiento de músculos oculares en tomografía computada (TC), compromiso sobre el 50 $\%$ del piso orbitario, hiperestesia progresiva del nervio maxilar (Burnstine, 2002; Gart \& Gosain, 2014). En los casos presentados, los principales signos que indicaron la necesidad de intervención quirúrgica fueron la presencia de diplopía y enoftalmos.

La pérdida de referencias anatómicas dificulta el posicionamiento de elementos para la reconstrucción orbitaria, dándose la posibilidad de un mal posicionamiento de estos. Lo anterior se puede traducir en una sobrecorrección del defecto o subcorrección, disminuyendo o aumentando el volumen orbitario respectivamente. Esto puede provocar secuelas funcionales como enoftalmos, exoftalmos y diplopía (Bell \& Markiewicz; Shaye et al.).Existen también complicaciones asociadas al implante utilizado en la reconstrucción, entre las que consideran extrusión, infección, palpabilidad y migración. La mayoría de las complicaciones en la reconstrucción orbitaria son atribuibles a una inadecuada reconstitución de los contornos óseos de la misma (Bell \& Markiewicz). Se suma además un mayor tiempo intraoperatorio principalmente asociado a lo complejo de dar una correcta adaptación a la malla de titanio.

El volumen aproximado de la cavidad orbitaria es de $30 \mathrm{ml}$, de los cuales $7 \mathrm{ml}$ estarían ocupados por el globo ocular (René, 2006). Algunos estudios proponen que un aumento del $5 \%$ en el volumen de la órbita podría causar enoftalmo clínicamente significativo (Bite et al., 1985; Whitehouse et al., 1994). Se ha reportado un aumento del volumen orbitario post reconstrucción en un $8.5 \%$ de los casos (Bell \& Markiewicz).
En caso de fracturas severas, la porción posterior del piso de la órbita puede ser inestable o no identificable, lo que dificulta el posicionamiento del implante y aumenta el riesgo de deficiencia funcional ocular. $\mathrm{Si}$ la proyección de implante queda muy alta, puede causar exoftalmos y problemas en la motilidad ocular, por otro lado, si el implante se ubica muy profundo en la órbita, podría afectar al nervio óptico causando pérdida de la visión del ojo afectado (Boyette et al., 2015).

El uso de tecnología digital como el estudio de imágenes vía $\mathrm{TC}$, la creación de objetos digitales a través del diseño asistido por computadora (CAD) y de la manufactura asistida por computadora (CAM), se ha vuelto cada vez más común en distintas disciplinas médicas tanto con fines educativos como terapéuticos (Jacobs \& Lin, 2017).

Una tomografía computada de alta resolución preoperatoria, acompañada de planificación digital puede ayudar a prevenir resultados asimétricos(Rana et al., 2019).También el uso de imágenes intraoperatorias como la tomografía computada, brinda la ventaja de mostrar la posición específica del implante una vez instalado (Boyette et al.), evitando defectos en su moldeo o localización. En el tercer caso presentado, el uso de esta tecnología permitió confirmar de forma pertinente el adecuado posicionamiento tridimensional de la malla preformada sobre el sitio afectado, evitando así una futura reintervención de la paciente.

Además de brindar una visión digital de la lesión, los datos obtenidos vía TC pueden ser utilizados para generar objetos digitales que posteriormente permiten la impresión de modelos estéreolitográficos. En los casos presentados, la TC permitió realizar una valoración inicial de las fracturas y la obtención de modelos físicos, los que se utilizaron en una evaluación in situ de la arquitectura ósea a reparar y en el moldeado de los elementos protésicos. Dentro de las ventajas de la utilización de estos modelos están una mejor visualización de la anatomía del paciente y de la topografía de la lesión, y la posibilidad de modelar ex vivo el material protésico para reparar la lesión, resultando una mejor adaptación de estos (Boyette et al.).

En los casos presentados, el uso de estos modelos estereolitográficos, ya fuese de forma convencional o con imagen en espejo de la órbita sana, permitió una disminución del tiempo operatorio, ya que la malla estuvo preformada al momento de la cirugía, logrando así un mejor asentamiento en el sitio defectuoso al ser posicionada, y evitando además un exce- 
so de fuerzas adicionales de flexión sobre el material protésico, las cuales pueden debilitarlo volviéndolo propenso a la fractura.

\section{CONCLUSIÓN}

El uso de tecnologías tridimensionales en la reparación de fracturas orbitaria es de gran ayuda para el cirujano, permitiendo dar una visión previa de la reparación quirúrgica, disminuyendo el tiempo intraoperatorio utilizado en el ajuste de los elementos protésicos, aumentando el éxito en su posicionamiento tridimensional y disminuyendo la morbilidad quirúrgica en el paciente.

SOTO, G. R.; ASTUDILLO, R. M.; BRAVO, A. R. \& GUNCKEL, M. R. Orbital fracture reconstruction using digital three-dimensional technology: a way to optimize results. Int. J. Odontostomat., 15(3):782-787, 2021.

ABSTRACT: The reconstruction of fractured orbital walls is complex due to the many volumetric parameters involved. An inadequate restitution of these walls may be associated with postsurgical sequelae in the patient. Is vitally important to count with tools that optimize the restitution of the orbit's anatomic shape during its reconstruction, and the use of new technologies has allowed the improvement of the surgical results, both anatomical and functional. The aim of this article is to show two surgical tools that allow to optimize the therapeutic results in patients with orbital fracture, which are stereolithographic models with mirror image technique, and intraoperative computed tomography. Their characteristics, their use in three cases of patients with orbital fractures, and the postoperative results are shown.

KEY WORDS: Orbital fracture, Three-dimensional printing (3D printing), stereolithography, computed tomography.

\section{REFERENCIAS BIBLIOGRÁFICAS}

Bell, R. B. \& Markiewicz, M. R. Computer-assisted planning, stereolithographic modeling, and intraoperative navigation for complex orbital reconstruction: a descriptive study in a preliminary cohort. J. Oral Maxillofac. Surg., 67(12):2559-70, 2009.

Bite, U.; Jackson, I. T.; Forbes, G. S. \& Gehring, D. G. Orbital volume measurements in enophthalmos using three-dimensional CT imaging. Plast. Reconstr. Surg., 75(4):502-7, 1985.

Boyette, J. R.; Pemberton, J. D. \& Bonilla-Velez, J. Management of orbital fractures: challenges and solutions. Clin. Ophthalmol., 9:2127-37, 2015.
Burnstine, M. A. Clinical recommendations for repair of isolated orbital floor fractures: An evidence-based analysis. Ophthalmology, 109(7):1207-10, 2002.

Gart, M. S. \& Gosain, A. K. Evidence-based medicine: Orbital floor fractures. Plast. Reconstr. Surg.,134(6):1345e-55e, 2014.

Ghai, S.; Sharma, Y.; Jain, N.; Satpathy, M. \& Pillai, A. K. Use of 3$D$ printing technologies in craniomaxillofacial surgery: a review. Oral Maxillofac. Surg., 22(3):249-59, 2018.

Jacobs, C. A. \& Lin, A. Y. A new classification of three-dimensional printing technologies: Systematic review of three-dimensional printing for patient-specific craniomaxillofacial surgery. Plast. Reconstr., 139(5):1211-20, 2017.

Rana, M.; Holtmann, H.; Kanatas, A. N.; Singh, D. D.; Sproll, C. K.; Kübler, N. R.; Ipaktchi, R.; Hufendiek, K. \& Gellrich, N. C. Primary orbital reconstruction with selective laser melted core patientspecific implants: overview of 100 patients. Br. J. Oral Maxillofac. Surg., 57(8):782-7, 2019.

René, C. Update on orbital anatomy. Eye (Lond.), 20(10):1119-29, 2006.

Shaye, D. A.; Tollefson, T. T. \& Strong, E. B. Use of intraoperative computed tomography for maxillofacial reconstructive surgery. JAMA Facial Plast. Surg., 17(2):113-9, 2015.

Warwar, R. E.; Bullock, J. D.; Ballal, D. R. \& Ballal, R. D. Mechanisms of orbital floor fractures: A clinical, experimental, and theoretical study. Ophthal. Plast. Reconstr. Surg., 16(3):188-200, 2000.

Whitehouse, R. W.; Batterbury, M.; Jackson, A. \& Noble, J. L. Prediction of enophthalmos by computed tomography after "blow out" orbital fracture. Br. J. Ophthalmol., 78(8):618-20, 1994.

Dirección para correspondencia:

Rubén Soto Galaz

Olivos 943

Independencia

Región Metropolitana

Santiago

CHILE

E-mail: ruben.soto@odontologia.uchile.cl 